\title{
SIDIS in the target fragmentation region: polarized and transverse momentum dependent fracture functions
}

\author{
M. Anselmino ${ }^{a}$, V. Barone ${ }^{b}$, A. Kotzinian ${ }^{a, c}$ \\ ${ }^{a}$ Dipartimento di Fisica Teorica, Università di Torino; \\ INFN, Sezione di Torino, 10125 Torino, Italy \\ ${ }^{b}$ Di.S.T.A., Università del Piemonte Orientale "A. Avogadro"; \\ INFN, Gruppo Collegato di Alessandria, 15121 Alessandria, Italy \\ ${ }^{c}$ Yerevan Physics Institute, 375036 Yerevan, Armenia
}

\begin{abstract}
The target fragmentation region of semi-inclusive deep inelastic scattering is described at leading twist, taking beam and target polarizations into account. The formalism of polarized and transversemomentum dependent fracture functions is developed and the observables for some specific processes are presented.
\end{abstract}

Key words: Semi-inclusive DIS, Target Fragmentation, Fracture Functions, Polarization, Transverse Momentum 


\section{Introduction}

The study of the 3-dimensional partonic structure of nucleons has become in recent years a central issue in hadron physics, with impressive dedicated theoretical and experimental activities. The ultimate goal is that of achieving a 3-dimensional imaging of the nucleons, both in configuration and momentum space. The information on the momentum distributions of quarks and gluons is encoded in the Transverse Momentum Dependent distribution functions (TMDs), which are probed in inclusive processes, mainly in Semi Inclusive Deep Inelastic Scattering (SIDIS, $l N \rightarrow l h X$ ).

In SIDIS TMDs can be accessed (at leading order in $\alpha_{Q E D}$ ) studying the azimuthal modulations of the cross section around the virtual photon direction (for a recent review see Ref. [1]). The detected final hadron is generated in the fragmentation of a scattered quark, the so-called Current Fragmentation Region (CFR). The cross section is then factorized as a convolution of transverse momentum dependent distribution and fragmentation functions; thus, in order to explore the parton momentum structure of the nucleon one has to be sure to select events in the CFR.

However, final hadrons in SIDIS and other partially inclusive processes can also be found among the remnants of the struck target, the so-called Target Fragmentation Region (TFR). The appropriate QCD formalism developed to study particle production in the TFR is that of the Fracture Functions, introduced by Trentadue and Veneziano [2] to describe the partonic structure of a nucleon when it fragments into a final-state hadron.

Although, in principle, the two regions can be kinematically separated, some situations may not be so clear; in particular at intermediate energies, when the total number of final produced particles is not very high. It is then worth studying the properties and the azimuthal distribution of hadrons produced in the TFR. This could both serve as a test of our complete understanding of the different mechanisms in the SIDIS production of hadrons and add additional information on the features of hadrons produced in the two regions. In some cases the same azimuthal dependences can be found in the CFR and in the TFR; then, great care must be taken when analyzing the data. Some other azimuthal dependences, instead, are typical and unique for one of the two regions, strengthening their interpretation.

Let us briefly recall how the production mechanisms in the two regions, CFR and TFR, can be described in semi-inclusive deep inelastic scattering (SIDIS), $l(\ell)+N(P) \rightarrow l\left(\ell^{\prime}\right)+h\left(P_{h}\right)+X\left(P_{X}\right)$. Its kinematics is described by the usual invariants $\left(q^{\mu}\right.$ is the momentum of the exchanged virtual photon)

$$
x_{B}=\frac{Q^{2}}{2 P \cdot q} \quad y=\frac{P \cdot q}{P \cdot \ell} \quad z_{h}=\frac{P \cdot P_{h}}{P \cdot q} \quad W^{2}=(P+q)^{2} .
$$

In the center of mass frame of the virtual photon and the nucleon (the c.m. $\gamma^{*} N$ frame), with the photon directed along the positive $z$ direction, the variable $z_{h}$ is

$$
z_{h} \simeq \frac{P_{h}^{+}}{q^{+}}
$$

where the light-cone components of a generic vector $A^{\mu}$ are defined as $A^{ \pm} \equiv\left(A^{0} \pm A^{3}\right) / \sqrt{2}$. In the ratio (2) $q^{+}$is always large, $q^{+} \sim Q$, whereas the magnitude of $P_{h}^{+}$determines the fragmentation region: 

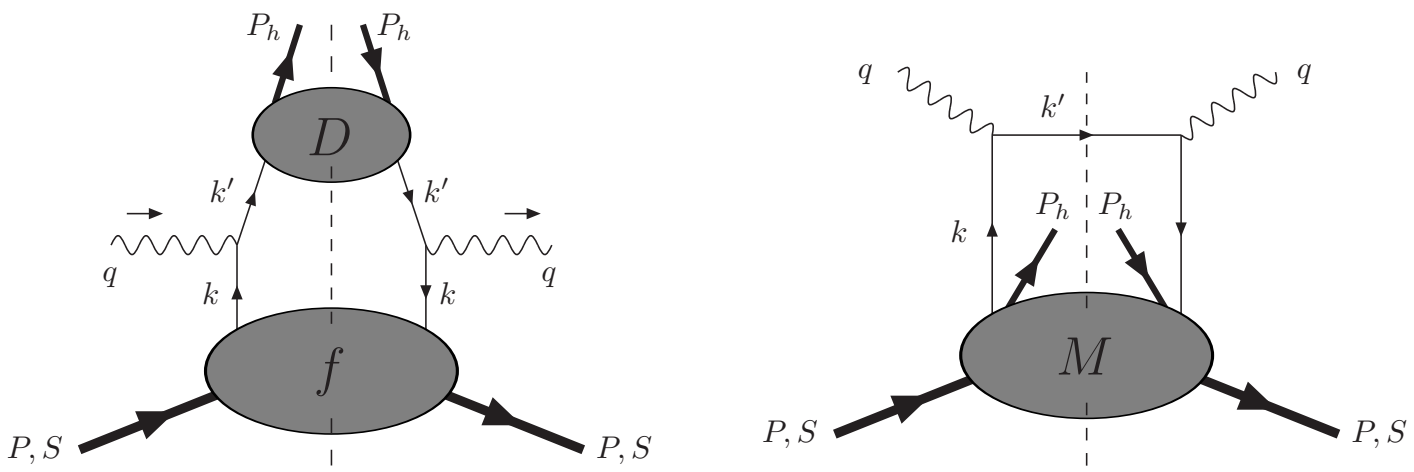

Figure 1: The handbag diagram for the SIDIS hadronic tensor in the current fragmentation region (left) and in the target fragmentation region (right).

Current fragmentation region (CFR): $P_{h}^{+} \sim Q$, Target fragmentation region $(\mathrm{TFR}): \quad P_{h}^{+} \sim 0$.

Equivalently, defining in the c.m. $\gamma^{*} N$ frame the hadron momentum as $P_{h}=\left(E_{h}, \boldsymbol{P}_{h \perp}, P_{h \|}\right)$, the usual Feynman variable $x_{F}=2 P_{h \|} / W$ identifies the CFR and the TFR, respectively, by $x_{F}>0$ and $x_{F}<0$. A detailed discussion of the operational criteria to separate the two regions can be found in Ref. [3].

In the CFR the SIDIS cross section integrated over the transverse momentum of the final hadron can be factorized at lowest order as

$$
\frac{\mathrm{d} \sigma^{\mathrm{CFR}}}{\mathrm{d} x_{B} \mathrm{~d} y \mathrm{~d} z_{h}}=\sum_{a} e_{a}^{2} f_{a}\left(x_{B}\right) \frac{\mathrm{d} \hat{\sigma}}{\mathrm{d} y} D_{a}\left(z_{h}\right),
$$

where $f_{a}\left(x_{B}\right)$ is the distribution function of parton $a, D_{a}\left(z_{h}\right)$ is the fragmentation function of parton $a$ into hadron $h$ and $\mathrm{d} \hat{\sigma} / \mathrm{d} y$ is the elementary cross section of lepton-quark scattering. The partonmodel graph describing this process is the handbag diagram shown in Fig. 1 (left). The partonic meaning of the two variables is the following: $x_{B}$ is the fraction of the longitudinal momentum of the nucleon carried by the quark, $z_{h}$ is the fraction of the longitudinal momentum of the struck quark carried by the final hadron (we have dropped all scale dependences in the distribution and fragmentation functions).

In the TFR the factorization in $x_{B}$ and $z_{h}$ of Eq. (3) does not hold any longer, as it is not possible to separate the quark emission from the hadron production. Moreover, $z_{h}$ is not the proper variable to describe this region. The reason is easily understood if we write $z_{h}$ in the c.m. $\gamma^{*} N$ frame (we neglect as usual hadron masses):

$$
z_{h}=\frac{E_{h}}{E\left(1-x_{B}\right)} \frac{\left(1-\cos \theta_{h}\right)}{2}
$$

where $\theta_{h}$ is the angle between $\boldsymbol{P}_{h}$ and $\boldsymbol{P}$. The $z_{h}$ variable does not discriminate between two different 
physical situations, namely $E_{h}=0$ (soft hadron emission) and $\theta_{h}=0$ (target fragmentation: emission of a hadron collinear with the target remnant), which both correspond to $z_{h}=0$.

In order to describe the production of hadrons in the target fragmentation region, one has to define the fracture functions $M_{a}\left(x_{B},\left(1-x_{B}\right) z\right)$, which depend on $x_{B}$ and on a new variable $z=E_{h} / E(1-$ $x_{B}$ ), and represent the distributions of partons inside a nucleon fragmenting almost collinearly into a given hadron [4, 5]. Notice that, differently from $z_{h}$, the variable $z$ vanishes in the soft limit only $\left(E_{h} \rightarrow 0\right)$. The SIDIS cross section in the TFR, integrated over the transverse momentum of the final hadron, thus becomes

$$
\frac{\mathrm{d} \sigma^{\mathrm{TFR}}}{\mathrm{d} x_{B} \mathrm{~d} y \mathrm{~d} z}=\sum_{a} e_{a}^{2}\left(1-x_{B}\right) M_{a}\left(x_{B},\left(1-x_{B}\right) z\right) \frac{\mathrm{d} \hat{\sigma}}{\mathrm{d} y} .
$$

In the following we will consider SIDIS processes in the TFR and will develop the formalism of fracture functions for polarized SIDIS and for $\boldsymbol{P}_{h \perp}$ distributions. We will introduce and classify all the leading-twist transverse momentum dependent and polarized fracture functions and present the lowest-order results for cross sections and angular distributions of single-hadron lepto-production with polarized beam and/or target.

\section{SIDIS in the target fragmentation region}

The SIDIS cross section is, in the one photon exchange approximation, given by the contraction of a leptonic tensor $L^{\mu \nu}$ with the hadronic tensor $W^{\mu \nu}$ incorporating the structure of the target nucleon and the dynamics of fragmentation:

$$
2 E_{h} \frac{\mathrm{d} \sigma}{\mathrm{d} x_{B} \mathrm{~d} y \mathrm{~d}^{3} \boldsymbol{P}_{h}}=\frac{\pi \alpha_{\mathrm{em}}^{2} y}{Q^{4}} L_{\mu \nu} W^{\mu \nu} .
$$

It is convenient to use a Sudakov parametrization of the relevant momenta. We introduce two null vectors, $p^{\mu}$ and $n^{\mu}$, with $p \cdot n=1, p^{-}=P^{-}, n^{+}=1 / P^{-}, p^{+}=n^{-}=0$, and we work in a $\gamma^{*} N$ frame. The unit vector $\hat{\boldsymbol{q}} \equiv \boldsymbol{q} /|\boldsymbol{q}|$ identifies the positive $z$ direction. In terms of the Sudakov vectors $p^{\mu}$ and $n^{\mu}$ the four-momenta at hand are:

$$
\begin{aligned}
& P^{\mu}=p^{\mu}+\frac{m_{N}^{2}}{2} n^{\mu} \simeq p^{\mu} \\
& q^{\mu} \simeq \frac{Q^{2}}{2 x_{B}} n^{\mu}-x_{B} p^{\mu} \\
& P_{h}^{\mu}=\zeta p^{\mu}+\frac{\boldsymbol{P}_{h \perp}^{2}+m_{h}^{2}}{2 \zeta} n^{\mu}+P_{h \perp}^{\mu} \simeq \zeta p^{\mu}+P_{h \perp}^{\mu} .
\end{aligned}
$$

The momentum of the hadron is identified by the light-cone ratio $\zeta=P_{h}^{-} / P^{-} \simeq E_{h} / E=z(1-$ $\left.x_{B}\right)$ and its transverse component $P_{h \perp}^{\mu}=\left(0, \boldsymbol{P}_{h \perp}, 0\right)$, with an azimuthal angle $\phi_{h}$ in the plane perpendicular to the $\gamma^{*} N$ axis. Note that in the high-energy limit one has $\zeta \simeq P_{h \|} / P_{\|} \equiv x_{L} \simeq$ $\left(1-x_{B}\right)\left|x_{F}\right|$. Replacing $\boldsymbol{P}_{h}$ with the variables $\left(\zeta, \boldsymbol{P}_{h \perp}\right)$ and allowing for target polarization (see Ref. [6]), the cross section takes the form

$$
\frac{\mathrm{d} \sigma}{\mathrm{d} x_{B} \mathrm{~d} y \mathrm{~d} \zeta \mathrm{d}^{2} \boldsymbol{P}_{h \perp} \mathrm{d} \phi_{S}}=\frac{\alpha_{\mathrm{em}}^{2}}{4 Q^{4}} \frac{y}{\zeta} L_{\mu \nu} W^{\mu \nu} .
$$


Here $\phi_{S}$ is the azimuthal angle of the transverse component of $S^{\mu}$, the nucleon spin vector, parametrized as

$$
S^{\mu}=S_{\|} \frac{p^{\mu}}{m_{N}}-S_{\|} \frac{m_{N}}{2} n^{\mu}+S_{\perp}^{\mu} \simeq S_{\|} \frac{p^{\mu}}{m_{N}}+S_{\perp}^{\mu}
$$

A similar decomposition holds for the spin vector of the produced hadron $h$ :

$$
S_{h}^{\mu}=S_{h \|} \frac{\zeta p^{\mu}}{m_{h}}-S_{h \|} \frac{m_{h}}{2 \zeta} n^{\mu}+S_{h \perp}^{\mu} \simeq S_{\|} \frac{\zeta p^{\mu}}{m_{h}}+S_{h \perp}^{\mu}
$$

Strictly speaking one should distinguish between transverse vectors with respect to $\boldsymbol{P}$ and with respect to $\boldsymbol{P}_{h}$. However, this difference can be ignored as far as one neglects subleading corrections in $P^{-}$(i.e., higher twists).

The explicit expression of the symmetric part of the leptonic tensor in the $\gamma^{*} N$ frame is [7]

$$
\begin{aligned}
L_{(\mathrm{s})}^{\mu \nu}= & \frac{Q^{2}}{y^{2}}\left\{-2\left(1-y+\frac{y^{2}}{2}\right) g_{\perp}^{\mu \nu}+4(1-y)\left[\frac{x_{B}^{2}}{Q^{2}} p^{\mu} p^{\nu}+\frac{Q^{2}}{4 x_{B}^{2}} n^{\mu} n^{\nu}+\frac{1}{2} p^{\{\mu} n^{\nu\}}\right]\right. \\
& \left.+4(1-y)\left(\hat{\ell}_{\perp}^{\mu} \hat{\ell}_{\perp}^{\nu}+\frac{1}{2} g_{\perp}^{\mu \nu}\right)+2(2-y) \sqrt{1-y}\left[\frac{x_{B}}{Q} p^{\{\mu} \hat{\ell}_{\perp}^{\nu\}}+\frac{Q}{2 x_{B}} n^{\{\mu} \hat{\ell}_{\perp}^{\nu\}}\right]\right\},
\end{aligned}
$$

where $\ell_{\perp}^{\mu}$ is the transverse component of the incoming and outgoing lepton momentum $\left(\hat{\ell}_{\perp}^{\mu}=\ell_{\perp}^{\mu} /\left|\ell_{\perp}\right|\right)$, and $g_{\perp}^{\mu \nu}=g^{\mu \nu}-\left(p^{\mu} n^{\nu}+p^{\nu} n^{\mu}\right)$. The antisymmetric part of the leptonic tensor reads $\left(\lambda_{l}\right.$ is the helicity of the lepton and $\left.\epsilon_{\perp}^{\mu \nu} \equiv \epsilon^{\mu \nu \rho \sigma} p_{\rho} n_{\sigma}\right)$

$$
L_{(\mathrm{a})}^{\mu \nu}=\frac{Q^{2}}{y^{2}}\left\{-\mathrm{i} \lambda_{l} y(2-y) \epsilon_{\perp}^{\mu \nu}-2 \mathrm{i} \lambda_{l} y \sqrt{1-y} \epsilon^{\mu \nu \rho \sigma}\left(\frac{x_{B}}{Q} p_{\rho}-\frac{Q}{2 x_{B}} n_{\rho}\right) \hat{\ell}_{\perp \sigma}\right\} .
$$

In the parton model, or equivalently at lowest order in QCD, the hadronic tensor in the target fragmentation region is represented by the handbag diagram of Fig. 1 (right) and reads (to simplify the presentation, we consider only quarks, the extension to antiquarks being straightforward):

$$
\begin{aligned}
W^{\mu \nu}= & \frac{1}{(2 \pi)^{4}} \sum_{a} e_{a}^{2} \sum_{X} \int \frac{\mathrm{d}^{3} \boldsymbol{P}_{X}}{(2 \pi)^{3} 2 E_{X}} \int \frac{\mathrm{d}^{4} k}{(2 \pi)^{4}} \int \frac{\mathrm{d}^{4} k^{\prime}}{(2 \pi)^{4}} 2 \pi \delta\left(k^{\prime 2}\right) \times \\
& {\left[\bar{u}\left(k^{\prime}\right) \gamma^{\mu} \phi\left(k, P, P_{h}\right)\right]^{*}\left[\bar{u}\left(k^{\prime}\right) \gamma^{\nu} \phi\left(k, P, P_{h}\right)\right] \times } \\
& (2 \pi)^{4} \delta^{4}\left(P-k-P_{h}-P_{X}\right)(2 \pi)^{4} \delta^{4}\left(k+q-k^{\prime}\right)
\end{aligned}
$$

where we have introduced the matrix elements of the quark field between the nucleon and the composite state of the hadron and the target remnant:

$$
\phi_{i}\left(k, P, P_{h}\right) \equiv\left\langle P_{h}, S_{h} ; X\left|\psi_{i}(0)\right| P, S\right\rangle .
$$

Let us now define the fracture matrix $\mathcal{M}$ representing the partonic structure of the nucleon target when it fragments into the final-state hadron:

$$
\begin{aligned}
\mathcal{M}_{i j}\left(k ; P, S ; P_{h}, S_{h}\right)= & \sum_{X} \int \frac{\mathrm{d}^{3} \boldsymbol{P}_{X}}{(2 \pi)^{3} 2 E_{X}} \int \frac{\mathrm{d}^{4} \xi}{(2 \pi)^{4}} \mathrm{e}^{\mathrm{i} k \cdot \xi} \times \\
& \left\langle P, S\left|\bar{\psi}_{j}(0)\right| P_{h}, S_{h} ; X\right\rangle\left\langle P_{h}, S_{h} ; X\left|\psi_{i}(\xi)\right| P, S\right\rangle .
\end{aligned}
$$


In QCD a Wilson line $\mathcal{W}$ connecting the quark fields must be inserted in order to ensure gauge invariance. The antiquark fracture matrix is obtained from (17) by replacing $\bar{\psi}_{j}$ with $\psi_{i}$, and $\psi_{i}$ with $\bar{\psi}_{j}$. In $\mathcal{M}, \psi$ and $\bar{\psi}$ we skip a flavor index $a$.

Using the definition (17), the hadronic tensor becomes

$$
W^{\mu \nu}=\sum_{a} e_{a}^{2} \int \frac{\mathrm{d}^{4} k}{(2 \pi)^{4}} 2 \pi \delta\left[(k+q)^{2}\right] \operatorname{Tr}\left[\mathcal{M} \gamma^{\mu}(\not k+\not q) \gamma^{\nu}\right]
$$

The quark momentum can be parametrized as

$$
k^{\mu}=x p^{\mu}+\frac{k^{2}+\boldsymbol{k}_{\perp}^{2}}{2 x} n^{\mu}+k_{\perp}^{\mu} \simeq x p^{\mu}+k_{\perp}^{\mu},
$$

with $x \equiv k^{-} / P^{-}$, and the delta function enforcing the on-shellness of the struck quark sets $x=x_{B}$ :

$$
\delta\left[(k+q)^{2}\right]=\frac{1}{2 P \cdot q} \delta\left(x_{B}-k^{-} / P^{-}\right) .
$$

The most general decomposition of $\mathcal{M}$ in a basis of Dirac matrices would contain terms proportional to $\mathbb{1}, \gamma^{\mu}, \gamma^{\mu} \gamma_{5}, \gamma_{5}, \sigma^{\mu \nu} \gamma_{5}$. We are interested in leading-twist fracture functions, i.e. in terms of $\mathcal{M}$ that are of order $\left(P^{-}\right)^{1}$. At this order, only the vector, axial and tensor components of $\mathcal{M}$ appear [8]:

$$
\mathcal{M}=\frac{1}{2}\left(\mathcal{V}_{\mu} \gamma^{\mu}+\mathcal{A}_{\mu} \gamma_{5} \gamma^{\mu}+\mathrm{i} \mathcal{T}_{\mu \nu} \sigma^{\mu \nu} \gamma_{5}\right)
$$

where the coefficients $\mathcal{V}^{\mu}, \mathcal{A}^{\mu}$ and $\mathcal{T}^{\mu \nu}$ contain various combinations of the vectors, or pseudo-vectors, $P^{\mu}, P_{h}^{\mu}, k^{\mu}, S^{\mu}$ and $S_{h}^{\mu}$. However, for lepto-production in the TFR, the structure of the quark current is such that only the vector and axial terms contribute at any twist. This can be explicitly shown using in (18) the identity

$$
\gamma^{\mu} \gamma^{\rho} \gamma^{\nu}=\left(g^{\mu \rho} g^{\nu \sigma}+g^{\mu \sigma} g^{\nu \rho}-g^{\mu \nu} g^{\rho \sigma}\right) \gamma_{\sigma}+\mathrm{i} \epsilon^{\mu \rho \nu \sigma} \gamma_{5} \gamma_{\sigma}
$$

which allows splitting $W^{\mu \nu}$ into a symmetric and an antisymmetric part:

$$
\begin{aligned}
W_{(\mathrm{s})}^{\mu \nu}= & \frac{1}{2 P \cdot q} \sum_{a} e_{a}^{2} \int \frac{\mathrm{d}^{4} k}{(2 \pi)^{3}} \delta\left(x_{B}-k^{-} / P^{-}\right)\left[\left(k^{\mu}+q^{\mu}\right) \operatorname{Tr}\left(\mathcal{M} \gamma^{\nu}\right)\right. \\
& \left.+\left(k^{\nu}+q^{\nu}\right) \operatorname{Tr}\left(\mathcal{M} \gamma^{\mu}\right)-g^{\mu \nu}\left(k_{\rho}+q_{\rho}\right) \operatorname{Tr}\left(\mathcal{M} \gamma^{\rho}\right)\right] \\
W_{(\mathrm{a})}^{\mu \nu}= & \frac{1}{2 P \cdot q} \sum_{a} e_{a}^{2} \int \frac{\mathrm{d}^{4} k}{(2 \pi)^{3}} \delta\left(x_{B}-k^{-} / P^{-}\right) \mathrm{i} \epsilon^{\mu \rho \nu \sigma}\left(k_{\rho}+q_{\rho}\right) \operatorname{Tr}\left(\mathcal{M} \gamma_{\sigma} \gamma_{5}\right) .
\end{aligned}
$$

One then sees that the hadronic tensor contains $\mathcal{V}^{\mu}=\frac{1}{2} \operatorname{Tr}\left(\mathcal{M} \gamma^{\mu}\right)$ and $\mathcal{A}^{\mu}=\frac{1}{2} \operatorname{Tr}\left(\mathcal{M} \gamma^{\mu} \gamma_{5}\right)$ only. The absence of a tensor term in $\mathcal{M}$ means that single-particle lepto-production does not probe any fracture function of transversely polarised quarks: this is easily understood by looking at the handbag diagram for target fragmentation, which cannot flip the helicity of the struck quark. In order to observe the transverse polarisation of quarks, which is described by chirally-odd fracture functions, one needs to detect a second hadron in the CFR, in coincidence with the one in the TFR. 


\section{Leading-twist polarized and transverse-momentum depen- dent fracture functions}

The polarized and transverse-momentum dependent fracture functions appear in the expansion of the leading twist projections $\left(\Gamma=\gamma^{-}, \gamma^{-} \gamma_{5}, \mathrm{i} \sigma^{i-} \gamma_{5}\right)$

$$
\begin{aligned}
& \mathcal{M}^{[\Gamma]}\left(x_{B}, \boldsymbol{k}_{\perp}, \zeta, \boldsymbol{P}_{h \perp}\right) \\
& \equiv \frac{1}{4 \zeta} \int \frac{\mathrm{d} k^{+} \mathrm{d} k^{-}}{(2 \pi)^{3}} \delta\left(k^{-}-x_{B} P^{-}\right) \operatorname{Tr}(\mathcal{M} \Gamma) \\
&= \frac{1}{4 \zeta} \int \frac{\mathrm{d} \xi^{+} \mathrm{d}^{2} \boldsymbol{\xi}_{\perp}}{(2 \pi)^{6}} \mathrm{e}^{\mathrm{i}\left(x_{B} P^{-} \xi^{+-} \boldsymbol{k}_{\perp} \cdot \boldsymbol{\xi}_{\perp}\right)} \sum_{X} \int \frac{\mathrm{d}^{3} \boldsymbol{P}_{X}}{(2 \pi)^{3} 2 E_{X}} \times \\
&\left\langle P, S|\bar{\psi}(0) \Gamma| P_{h}, S_{h} ; X\right\rangle\left\langle P_{h}, S_{h} ; X\left|\psi\left(\xi^{+}, 0, \boldsymbol{\xi}_{\perp}\right)\right| P, S\right\rangle .
\end{aligned}
$$

These represent the conditional probabilities to find an unpolarized $\left(\Gamma=\gamma^{-}\right)$, a longitudinally polarized $\left(\Gamma=\gamma^{-} \gamma_{5}\right)$ or a transversely polarized $\left(\Gamma=\mathrm{i} \sigma^{i-} \gamma_{5}\right)$ quark with longitudinal momentum fraction $x_{B}$ and transverse momentum $\boldsymbol{k}_{\perp}$ inside a nucleon fragmenting into a hadron carrying a fraction $\zeta$ of the nucleon longitudinal momentum and a transverse momentum $\boldsymbol{P}_{h \perp}$. Again, in QCD a Wilson line $\mathcal{W}$ must be inserted, which for $\boldsymbol{k}_{\perp}$-dependent distributions includes transverse links and is generally rather complicated [9, 10]: its explicit structure, however, is irrelevant for our purposes.

The most general parameterization of the traced fracture matrix (25) can be written as:

$$
\begin{aligned}
\mathcal{M}^{\left[\gamma^{-}\right]}= & \hat{M}+\frac{\boldsymbol{P}_{h \perp} \times \boldsymbol{S}_{\perp}}{m_{h}} \hat{M}_{T}^{h}+\frac{\boldsymbol{k}_{\perp} \times \boldsymbol{S}_{\perp}}{m_{N}} \hat{M}_{T}^{\perp}+\frac{S_{\|}\left(\boldsymbol{k}_{\perp} \times \boldsymbol{P}_{h \perp}\right)}{m_{N} m_{h}} \hat{M}_{L}^{\perp h} \\
\mathcal{M}^{\left[\gamma^{-} \gamma_{5}\right]}= & S_{\|} \Delta \hat{M}_{L}+\frac{\boldsymbol{P}_{h \perp} \cdot \boldsymbol{S}_{\perp}}{m_{h}} \Delta \hat{M}_{T}^{h}+\frac{\boldsymbol{k}_{\perp} \cdot \boldsymbol{S}_{\perp}}{m_{N}} \Delta \hat{M}_{T}^{\perp}+\frac{\boldsymbol{k}_{\perp} \times \boldsymbol{P}_{h \perp}}{m_{N} m_{h}} \Delta \hat{M}^{\perp h} \\
\mathcal{M}^{\left[\mathrm{i} \sigma^{i-} \gamma_{5}\right]}= & S_{\perp}^{i} \Delta_{T} \hat{M}_{T}+\frac{S_{\|} P_{h \perp}^{i}}{m_{h}} \Delta_{T} \hat{M}_{L}^{h}+\frac{S_{\|} k_{\perp}^{i}}{m_{N}} \Delta_{T} \hat{M}_{L}^{\perp} \\
& +\frac{\left(\boldsymbol{P}_{h \perp} \cdot \boldsymbol{S}_{\perp}\right) P_{h \perp}^{i}}{m_{h}^{2}} \Delta_{T} \hat{M}_{T}^{h h}+\frac{\left(\boldsymbol{k}_{\perp} \cdot \boldsymbol{S}_{\perp}\right) k_{\perp}^{i}}{m_{N}^{2}} \Delta_{T} \hat{M}_{T}^{\perp \perp} \\
& +\frac{\left(\boldsymbol{k}_{\perp} \cdot \boldsymbol{S}_{\perp}\right) P_{h \perp}^{i}-\left(\boldsymbol{P}_{h \perp} \cdot \boldsymbol{S}_{\perp}\right) k_{\perp}^{i}}{m_{N} m_{h}} \hat{M}_{T}^{\perp h} \\
& +\frac{\epsilon_{\perp}^{i j} P_{h \perp j}}{m_{h}} \Delta_{T} \hat{M}^{h}+\frac{\epsilon_{\perp}^{i j} k_{\perp j}}{m_{N}} \Delta_{T} \hat{M}^{\perp}
\end{aligned}
$$

where by the vector product of the two-dimensional vectors we mean the pseudo-scalar quantity $\boldsymbol{a}_{\perp} \times \boldsymbol{b}_{\perp}=\epsilon_{\perp i j} a_{\perp}^{i} b_{\perp}^{j}=\left|\boldsymbol{a}_{\perp}\right|\left|\boldsymbol{b}_{\perp}\right| \sin \left(\phi_{b}-\phi_{a}\right)$. All fracture functions depend on the scalar variables $x_{B}, \boldsymbol{k}_{\perp}^{2}, \zeta, \boldsymbol{P}_{h \perp}^{2}, \boldsymbol{k}_{\perp} \cdot \boldsymbol{P}_{h \perp}$. An important point to notice is that while parity invariance constrains the structure of the fracture matrix, time reversal invariance does not, since $\mathcal{M}$, similarly to the fragmentation matrix, contains the out-states $\left|P_{h}, S_{h} ; X\right\rangle$. In fact the fracture functions of Eqs. (26)(28) can be seen to reflect the independent combinations, with the appropriate parity properties, of all vectors and pseudo-vectors at our disposal.

As most of the functions introduced above appear for the first time, a few words about them and some explanation of the notations adopted can be useful. We denote by $\hat{M}$ the unintegrated 
fracture functions of unpolarized quarks, by $\Delta \hat{M}$ the unintegrated fracture functions of longitudinally polarized quarks and by $\Delta_{T} \hat{M}$ the unintegrated fracture functions of transversely polarized quarks. The subscripts $L$ and $T$, appended to $\hat{M}$, label the polarization of the target (no subscript $=$ unpolarized, $L=$ longitudinally polarized, $T=$ transversely polarized). The superscripts $h$ and $\perp$ signal the presence of factors $P_{h \perp}^{i}$ and $k_{\perp}^{i}$, respectively. Fracture functions integrated over $\boldsymbol{k}_{\perp}$ will not have the hats.

- $\hat{M}$ is the unintegrated distribution of unpolarized quarks inside an unpolarized nucleon fragmenting into a spinless hadron emitted with a non-zero transverse momentum. Integrating $\hat{M}$ over $\boldsymbol{P}_{h \perp}$ and $\boldsymbol{k}_{\perp}$, one obtains the collinear fracture function introduced in Refs. [2, 4]:

$$
M\left(x_{B}, \zeta\right)=\int \mathrm{d}^{2} \boldsymbol{P}_{h \perp} \int \mathrm{d}^{2} \boldsymbol{k}_{\perp} \hat{M}\left(x_{B}, \boldsymbol{k}_{\perp}^{2}, \zeta, \boldsymbol{P}_{h \perp}^{2}, \boldsymbol{k}_{\perp} \cdot \boldsymbol{P}_{h \perp}\right) .
$$

- $\hat{M}_{T}^{h}$ and $\hat{M}_{T}^{\perp}$ are new fracture functions describing the distributions of unpolarized quarks inside a transversely polarized target. If we integrate $\mathcal{M}^{\left[\gamma^{-}\right]}$over the quark transverse momentum by means of the identities in the Appendix, we are left with two $\boldsymbol{P}_{h \perp}$-dependent fracture functions:

$$
\int \mathrm{d}^{2} \boldsymbol{k}_{\perp} \mathcal{M}^{\left[\gamma^{-}\right]}=M\left(x_{B}, \zeta, \boldsymbol{P}_{h \perp}^{2}\right)+\frac{\boldsymbol{P}_{h \perp} \times \boldsymbol{S}_{\perp}}{m_{h}} M_{T}^{h}\left(x_{B}, \zeta, \boldsymbol{P}_{h \perp}^{2}\right),
$$

where

$$
M\left(x_{B}, \zeta, \boldsymbol{P}_{h \perp}^{2}\right)=\int \mathrm{d}^{2} \boldsymbol{k}_{\perp} \hat{M}\left(x_{B}, \boldsymbol{k}_{\perp}^{2}, \zeta, \boldsymbol{P}_{h \perp}^{2}, \boldsymbol{k}_{\perp} \cdot \boldsymbol{P}_{h \perp}\right)
$$

was called "extended fracture function" in Ref. [11]. $M_{T}^{h}$ is obtained from a combination of two unintegrated fracture functions:

$$
M_{T}^{h}\left(x_{B}, \zeta, \boldsymbol{P}_{h \perp}^{2}\right)=\int \mathrm{d}^{2} \boldsymbol{k}_{\perp}\left\{\hat{M}_{T}^{h}+\frac{m_{h}}{m_{N}} \frac{\boldsymbol{k}_{\perp} \cdot \boldsymbol{P}_{h \perp}}{\boldsymbol{P}_{h \perp}^{2}} \hat{M}_{T}^{\perp}\right\} .
$$

Notice that only the correlation $\boldsymbol{P}_{h \perp} \times \boldsymbol{S}_{\perp}=\left|\boldsymbol{P}_{h \perp}\right|\left|\boldsymbol{S}_{\perp}\right| \sin \left(\phi_{S}-\phi_{h}\right)$ survives upon integration over $\boldsymbol{k}_{\perp}$.

- $\hat{M}_{L}^{\perp h}$, the last independent fracture function contributing to $\mathcal{M}^{\left[\gamma^{-}\right]}$, describes the distribution of unpolarized quarks in a longitudinally polarized nucleon. It can only exist thanks to the scalar combination $S_{\|}\left(\boldsymbol{k}_{\perp} \times \boldsymbol{P}_{h \perp}\right)$ and does not survive upon integration.

- Turning to $\mathcal{M}^{\left[\gamma^{-} \gamma^{5}\right]}, \Delta \hat{M}_{L}$ is the unintegrated fracture function of longitudinally polarized quarks in a longitudinally polarized target; it yields the helicity fracture function $\Delta M_{L}\left(x_{B}, \zeta\right)$, once integrated over $\boldsymbol{k}_{\perp}$ and $\boldsymbol{P}_{h \perp}$.

- $\Delta \hat{M}_{T}^{h}, \Delta \hat{M}_{T}^{\perp}$ and $\Delta \hat{M}^{\perp h}$ are new fracture functions describing the distribution of longitudinally polarized quarks inside a transversely polarized nucleon (the first two) and an unpolarized nucleon $\left(\Delta \hat{M}^{\perp h}\right)$. Integrating $\mathcal{M}^{\left[\gamma^{-} \gamma_{5}\right]}$ over $\boldsymbol{k}_{\perp}$ with the help of the identities in the Appendix yields

$$
\int \mathrm{d}^{2} \boldsymbol{k}_{\perp} \mathcal{M}^{\left[\gamma^{-} \gamma_{5}\right]}=S_{\|} \Delta M_{L}\left(x_{B}, \zeta, \boldsymbol{P}_{h \perp}^{2}\right)+\frac{\boldsymbol{P}_{h \perp} \cdot \boldsymbol{S}_{\perp}}{m_{h}} \Delta M_{T}^{h}\left(x_{B}, \zeta, \boldsymbol{P}_{h \perp}^{2}\right),
$$


where $\Delta M_{L}$ is

$$
\Delta M_{L}\left(x_{B}, \zeta, \boldsymbol{P}_{h \perp}^{2}\right)=\int \mathrm{d}^{2} \boldsymbol{k}_{\perp} \Delta \hat{M}_{L}\left(x_{B}, \boldsymbol{k}_{\perp}^{2}, \zeta, \boldsymbol{P}_{h \perp}^{2}, \boldsymbol{k}_{\perp} \cdot \boldsymbol{P}_{h \perp}\right),
$$

and $\Delta M_{T}^{h}$ is related to two unintegrated fracture functions as follows

$$
\Delta M_{T}^{h}\left(x_{B}, \zeta, \boldsymbol{P}_{h \perp}^{2}\right)=\int \mathrm{d}^{2} \boldsymbol{k}_{\perp}\left\{\Delta \hat{M}_{T}^{h}+\frac{m_{h}}{m_{N}} \frac{\boldsymbol{k}_{\perp} \cdot \boldsymbol{P}_{h \perp}}{\boldsymbol{P}_{h \perp}^{2}} \Delta \hat{M}_{T}^{\perp}\right\} .
$$

In this case, the only surviving angular correlation is $\boldsymbol{P}_{h \perp} \cdot \boldsymbol{S}_{\perp}=\left|\boldsymbol{P}_{h \perp}\right|\left|\boldsymbol{S}_{\perp}\right| \cos \left(\phi_{S}-\phi_{h}\right)$.

- The fracture functions of transversely polarized quarks, contained in $\mathcal{M}^{\left[\mathrm{i} \sigma^{i-} \gamma_{5}\right]}$, are not probed in single-particle SIDIS - the process we are interested in here - and will be discussed in a separate paper devoted to two-hadron lepto-production 1 .

The inclusive lepto-production of a single spinless hadron in the TFR involves the integration over $\boldsymbol{k}_{\perp}$. It then probes, Eqs. (30) and (33), four fracture functions: $M, \Delta M_{L}, M_{T}^{h}, \Delta M_{T}^{h}$. These four fracture functions, giving the distribution of final hadrons inside polarized nucleons, can be seen as the analog of some TMDs, which are defined in the CFR. $M\left(x_{B}, \zeta, \boldsymbol{P}_{h \perp}^{2}\right)$ and $\Delta M_{L}\left(x_{B}, \zeta, \boldsymbol{P}_{h \perp}^{2}\right)$ correspond, respectively, to the unintegrated unpolarized and helicity distributions. $M_{T}^{h}\left(x_{B}, \zeta, \boldsymbol{P}_{h \perp}^{2}\right)$, which describes the distribution of unpolarized final hadrons $h$ inside a transversely polarized nucleon, is the TFR analog of the Sivers distribution function $f_{1 T}^{\perp}\left(x_{B}, \boldsymbol{k}_{\perp}^{2}\right)$ [14, 15], which describes the distribution of unpolarized quarks inside a transversely polarized nucleon (see Ref. [1] for a review of the theoretical and experimental work on the subject). $\Delta M_{T}^{h}\left(x_{B}, \zeta, \boldsymbol{P}_{h \perp}^{2}\right)$ is similar to the distribution function $g_{1 T}^{\perp}\left(x_{B}, \boldsymbol{k}_{\perp}^{2}\right)$, which describes longitudinally polarized quarks inside a transversely polarized nucleon [16, 17, 18].

Two important last general remarks are in order. First of all, the transverse momenta we are considering, $\boldsymbol{k}_{\perp}$ and $\boldsymbol{P}_{h \perp}$, are intended as intrinsic momenta, not generated by gluon radiation (this source of transverse momenta was explored by Ceccopieri and Trentadue [19, 20]). In other terms, the effects we are studying are of non-perturbative origin. Second, while the factorization theorem for collinear and $\boldsymbol{P}_{h \perp}$-dependent fracture functions has been proven [11, 21], no analogous result exists for fully unintegrated fracture functions. In this case, factorization is just an assumption.

\section{Momentum sum rules}

It is known [2] that the unpolarized collinear fracture function $M\left(x_{B}, \zeta\right)$ satisfies a momentum sum rule:

$$
\sum_{h} \int_{0}^{1-x_{B}} \mathrm{~d} \zeta \zeta M\left(x_{B}, \zeta\right)=\left(1-x_{B}\right) f_{1}\left(x_{B}\right)
$$

where $\sum_{h}$ is a sum over all hadrons, and $f_{1}$ is the ordinary number density of quarks. Equation (36) is easily understood by remembering the probabilistic interpretation of $M$ and noticing that, if the

\footnotetext{
${ }^{1}$ Some transversely polarized fracture functions have been considered by Sivers [12, 13, but their transverse momentum and transverse spin structure has not been explored.
} 
target emits a quark with momentum fraction $x_{B}$, the total momentum fraction available for the final hadron is $1-x_{B}$.

A set of generalized sum rules for the transverse-momentum dependent fracture functions introduced in the previous Section can be derived as follows. First of all, the integral over $\boldsymbol{P}_{X}$ and the $\sum_{X}$ of the out-states $\left|P_{h}, S_{h} ; X\right\rangle$ yields the number operator of the hadrons $h$ [22, 23]:

$$
\sum_{X} \int \frac{\mathrm{d}^{3} \boldsymbol{P}_{X}}{(2 \pi)^{3} 2 E_{X}}\left|P_{h}, S_{h} ; X\right\rangle\left\langle P_{h}, S_{h} ; X\right|=a_{h}^{\dagger} a_{h},
$$

where $a_{h}\left(a_{h}^{\dagger}\right)$ is the annihilation (creation) operator of the hadron $h$ with momentum $P_{h}$. The traced fracture matrix (25) can thus be rewritten as

$$
\mathcal{M}^{[\Gamma]}=\frac{1}{4 \zeta} \int \frac{\mathrm{d} \xi^{+} \mathrm{d}^{2} \boldsymbol{\xi}_{\perp}}{(2 \pi)^{6}} \mathrm{e}^{\mathrm{i}\left(x_{B} P^{-} \xi^{+-} \boldsymbol{k}_{\perp} \cdot \boldsymbol{\xi}_{\perp}\right)}\left\langle P, S\left|\bar{\psi}(0) \Gamma a_{h}^{\dagger} a_{h} \psi\left(\xi^{+}, 0, \boldsymbol{\xi}_{\perp}\right)\right| P, S\right\rangle .
$$

If we now multiply $\mathcal{M}^{[\Gamma]}$ by $\zeta$, integrate over $\zeta$ and $\boldsymbol{P}_{h \perp}$, and sum over all hadrons, we get

$$
\begin{aligned}
\sum_{h} \int_{0}^{1-x_{B}} \mathrm{~d} \zeta \zeta \int \mathrm{d}^{2} \boldsymbol{P}_{h \perp} \mathcal{M}^{[\Gamma]}\left(x_{B}, \boldsymbol{k}_{\perp}, \zeta, \boldsymbol{P}_{h \perp}\right) \\
\quad=\sum_{h} \int_{0}^{1-x_{B}} \mathrm{~d}\left(\frac{P_{h}^{-}}{P^{-}}\right) \frac{P_{h}^{-}}{P^{-}} \int \mathrm{d}^{2} \boldsymbol{P}_{h \perp} \mathcal{M}^{[\Gamma]}\left(x_{B}, \boldsymbol{k}_{\perp}, \zeta, \boldsymbol{P}_{h \perp}\right) \\
\quad=\frac{1}{2} \int \frac{\mathrm{d} \xi^{+} \mathrm{d}^{2} \boldsymbol{\xi}_{\perp}}{(2 \pi)^{3}} \mathrm{e}^{\mathrm{i}\left(x_{B} P^{-} \xi^{+-} \boldsymbol{k}_{\perp} \cdot \boldsymbol{\xi}_{\perp}\right)} \sum_{h} \int \frac{\mathrm{d} P_{h}^{-} \mathrm{d}^{2} \boldsymbol{P}_{h \perp}}{(2 \pi)^{3} 2 P_{h}^{-}}\left\langle P, S\left|\bar{\psi}(0) \Gamma \frac{P_{h}^{-}}{P^{-}} a_{h}^{\dagger} a_{h} \psi\left(\xi^{+}, 0, \boldsymbol{\xi}_{\perp}\right)\right| P, S\right\rangle \\
\quad=\left(1-x_{B}\right) \Phi^{[\Gamma]}\left(x_{B}, \boldsymbol{k}_{\perp}\right),
\end{aligned}
$$

where

$$
\Phi^{[\Gamma]}\left(x_{B}, \boldsymbol{k}_{\perp}\right)=\frac{1}{2} \int \frac{\mathrm{d} \xi^{+} \mathrm{d}^{2} \boldsymbol{\xi}_{\perp}}{(2 \pi)^{3}} \mathrm{e}^{\mathrm{i}\left(x_{B} P^{-} \xi^{+-}-\boldsymbol{k}_{\perp} \cdot \boldsymbol{\xi}_{\perp}\right)}\left\langle P, S\left|\bar{\psi}(0) \Gamma \psi\left(\xi^{+}, 0, \boldsymbol{\xi}_{\perp}\right)\right| P, S\right\rangle
$$

is the traced quark correlation matrix containing the transverse momentum dependendent distributions. Equation (39) establishes a relation between the fracture functions of Eqs. (26)-(28) and the TMDs appearing in the expansion of $\Phi^{\left[\gamma^{-}\right]}, \Phi^{\left[\gamma^{-} \gamma_{5}\right]}$ and $\Phi^{\left[\mathrm{i} \sigma^{i-} \gamma_{5}\right]}$ (for which see, e.g., Ref. [1]). The final results, obtained by using the identities of the Appendix, are

$$
\begin{aligned}
& \sum_{h} \int \mathrm{d} \zeta \zeta \int \mathrm{d}^{2} \boldsymbol{P}_{h \perp} \hat{M}=\left(1-x_{B}\right) f_{1}\left(x_{B}, \boldsymbol{k}_{\perp}^{2}\right) \\
& \sum_{h} \int \mathrm{d} \zeta \zeta \int \mathrm{d}^{2} \boldsymbol{P}_{h \perp}\left\{\hat{M}_{T}^{\perp}+\frac{m_{N}}{m_{h}} \frac{\boldsymbol{k}_{\perp} \cdot \boldsymbol{P}_{h \perp}}{\boldsymbol{k}_{\perp}^{2}} \hat{M}_{T}^{h}\right\}=-\left(1-x_{B}\right) f_{1 T}^{\perp}\left(x_{B}, \boldsymbol{k}_{\perp}^{2}\right) \\
& \sum_{h} \int \mathrm{d} \zeta \zeta \int \mathrm{d}^{2} \boldsymbol{P}_{h \perp} \Delta \hat{M}_{L}=\left(1-x_{B}\right) g_{1 L}\left(x_{B}, \boldsymbol{k}_{\perp}^{2}\right) \\
& \sum_{h} \int \mathrm{d} \zeta \zeta \int \mathrm{d}^{2} \boldsymbol{P}_{h \perp}\left\{\Delta \hat{M}_{T}^{\perp}+\frac{m_{N}}{m_{h}} \frac{\boldsymbol{k}_{\perp} \cdot \boldsymbol{P}_{h \perp}}{\boldsymbol{k}_{\perp}^{2}} \Delta \hat{M}_{T}^{h}\right\}=\left(1-x_{B}\right) g_{1 T}\left(x_{B}, \boldsymbol{k}_{\perp}^{2}\right) \\
& \sum_{h} \int \mathrm{d} \zeta \zeta \int \mathrm{d}^{2} \boldsymbol{P}_{h \perp}\left\{\Delta_{T} \hat{M}_{L}^{\perp}+\frac{m_{N}}{m_{h}} \frac{\boldsymbol{k}_{\perp} \cdot \boldsymbol{P}_{h \perp}}{\boldsymbol{k}_{\perp}^{2}} \Delta_{T} \hat{M}_{L}^{h}\right\}=\left(1-x_{B}\right) h_{1 L}^{\perp}\left(x_{B}, \boldsymbol{k}_{\perp}^{2}\right)
\end{aligned}
$$




$$
\begin{aligned}
& \sum_{h} \int \mathrm{d} \zeta \zeta \int \mathrm{d}^{2} \boldsymbol{P}_{h \perp}\left\{\Delta_{T} \hat{M}^{\perp}+\frac{m_{N}}{m_{h}} \frac{\boldsymbol{k}_{\perp} \cdot \boldsymbol{P}_{h \perp}}{\boldsymbol{k}_{\perp}^{2}} \Delta_{T} \hat{M}^{h}\right\}=-\left(1-x_{B}\right) h_{1}^{\perp}\left(x_{B}, \boldsymbol{k}_{\perp}^{2}\right) \\
& \sum_{h} \int \mathrm{d} \zeta \zeta \int \mathrm{d}^{2} \boldsymbol{P}_{h \perp}\left\{\Delta_{T} \hat{M}_{T}^{\perp \perp}+\frac{m_{N}^{2}}{m_{h}^{2}} \frac{2\left(\boldsymbol{k}_{\perp} \cdot \boldsymbol{P}_{h \perp}\right)^{2}-\boldsymbol{k}_{\perp}^{2} \boldsymbol{P}_{h \perp}^{2}}{\left(\boldsymbol{k}_{\perp}^{2}\right)^{2}} \Delta_{T} \hat{M}_{T}^{h h}\right\} \\
& \quad=\left(1-x_{B}\right) h_{1 T}^{\perp}\left(x_{B}, \boldsymbol{k}_{\perp}^{2}\right) \\
& \sum_{h} \int \mathrm{d} \zeta \zeta \int \mathrm{d}^{2} \boldsymbol{P}_{h \perp}\left\{\Delta_{T} \hat{M}_{T}+\frac{\boldsymbol{k}_{\perp}^{2}}{2 m_{N}^{2}} \Delta_{T} \hat{M}_{T}^{\perp \perp}+\frac{\boldsymbol{P}_{h \perp}^{2}}{2 m_{h}^{2}} \Delta_{T} \hat{M}_{T}^{h h}\right\}=\left(1-x_{B}\right) h_{1}\left(x_{B}, \boldsymbol{k}_{\perp}^{2}\right) .
\end{aligned}
$$

These are the momentum sum rules satisfied by the unintegrated fracture functions. They might be useful for constraining and guiding simple models of fracture functions.

\section{Cross sections and angular distributions}

Contracting the hadronic tensor, Eqs. (23), 24), with the symmetric and antisymmetric part of the leptonic tensor, Eqs. (13, 14), and using Eq. (25), yields

$$
\begin{aligned}
L_{(\mathbf{s})}^{\mu \nu} W_{\mu \nu}^{(\mathrm{s})} & =\frac{8 Q^{2}}{y^{2}}\left(1-y+\frac{y^{2}}{2}\right) \zeta \sum_{a} e_{a}^{2} \int \mathrm{d}^{2} \boldsymbol{k}_{\perp} \mathcal{M}^{\left[\gamma^{-}\right]} \\
L_{(\mathrm{a})}^{\mu \nu} W_{\mu \nu}^{(\mathrm{a})} & =\lambda_{l} \frac{8 Q^{2}}{y^{2}} y\left(1-\frac{y}{2}\right) \zeta \sum_{a} e_{a}^{2} \int \mathrm{d}^{2} \boldsymbol{k}_{\perp} \mathcal{M}^{\left[\gamma^{-} \gamma_{5}\right]} .
\end{aligned}
$$

We focus on three processes:

1. lepto-production of a spinless hadron, $l+N \rightarrow l^{\prime}+h+X$;

2. lepto-production of a spinless hadron plus a quark jet, $l+N \rightarrow l^{\prime}+h+$ jet $+X$;

3. lepto-production of a polarized hadron, $l+N \rightarrow l^{\prime}+h^{\uparrow}+X$ (integrated over all transverse momenta).

\subsection{Lepto-production of a spinless hadron}

Consider the lepto-production of an unpolarized or spinless hadron (for instance, pion lepto-production, which is the most common process). Inserting Eqs. (30, 331) into Eqs. (49, 501), and using Eq. (10), one finds that the cross section for this process is

$$
\begin{aligned}
& \frac{\mathrm{d} \sigma^{\mathrm{TFR}}}{\mathrm{d} x_{B} \mathrm{~d} y \mathrm{~d} \zeta \mathrm{d}^{2} \boldsymbol{P}_{h \perp} \mathrm{d} \phi_{S}}=\frac{2 \alpha_{\mathrm{em}}^{2}}{Q^{2} y}\left\{\left(1-y+\frac{y^{2}}{2}\right)\right. \\
& \quad \times \sum_{a} e_{a}^{2}\left[M\left(x_{B}, \zeta, \boldsymbol{P}_{h \perp}^{2}\right)-\left|\boldsymbol{S}_{\perp}\right| \frac{\left|\boldsymbol{P}_{h \perp}\right|}{m_{h}} M_{T}^{h}\left(x_{B}, \zeta, \boldsymbol{P}_{h \perp}^{2}\right) \sin \left(\phi_{h}-\phi_{S}\right)\right] \\
& +\lambda_{l} y\left(1-\frac{y}{2}\right) \sum_{a} e_{a}^{2}\left[S_{\|} \Delta M_{L}\left(x_{B}, \zeta, \boldsymbol{P}_{h \perp}^{2}\right)\right. \\
& \left.\left.+\left|\boldsymbol{S}_{\perp}\right| \frac{\left|\boldsymbol{P}_{h \perp}\right|}{m_{h}} \Delta M_{T}^{h}\left(x_{B}, \zeta, \boldsymbol{P}_{h \perp}^{2}\right) \cos \left(\phi_{h}-\phi_{S}\right)\right]\right\} .
\end{aligned}
$$


As already mentioned, this process is described by four fracture functions, two of which appear when the target is transversely polarized. The only azimuthal modulations arise from correlations between the transverse spin of the target and the transverse momentum of the final hadron, and are of the type $\sin \left(\phi_{h}-\phi_{S}\right)$ and $\cos \left(\phi_{h}-\phi_{S}\right)$ : the former manifests itself when the lepton beam is unpolarized, the latter, when the beam is longitudinally polarized.

It is interesting to compare our result with the corresponding situation in the current fragmentation region, where at leading twist there are six different modulations: besides the two which also appear in the TFR, that is $\sin \left(\phi_{h}-\phi_{S}\right)$ and $\cos \left(\phi_{h}-\phi_{S}\right)$, there is a $\cos 2 \phi_{h}$ term associated with the Boer-Mulders effect in unpolarized SIDIS [24, 25, 26], a $\sin 2 \phi_{h}$ term for a longitudinally polarized target, and, in the transversely polarized case, the $\sin \left(\phi_{h}+\phi_{S}\right)$ term, which gives access to the transversity distribution [27, 28], and the $\sin \left(3 \phi_{h}-\phi_{S}\right)$ term involving the distribution $h_{1 T}^{\perp}$. All these additional asymmetries arise due to the Collins effect in the fragmentation of a transversely polarized quark. The absence of these type of angular distributions in the TFR might have some relevance in phenomenological analyses.

Referring to the general parametrization of the SIDIS cross section presented in Ref. [29], $M_{T}^{h}$ and $\Delta M_{T}^{h}$ in (51) contribute, in the TFR, to the structure functions $F_{U T, T}^{\sin \left(\phi_{h}-\phi_{S}\right)}$ and $F_{L T}^{\cos \left(\phi_{h}-\phi_{S}\right)}$, respectively:

$$
\begin{aligned}
& {\left[F_{U T, T}^{\sin \left(\phi_{h}-\phi_{S}\right)}\right]_{\mathrm{TFR}}=-\sum_{a} e_{a}^{2} x_{B} \frac{\left|\boldsymbol{P}_{h \perp}\right|}{m_{h}} M_{T}^{h}\left(x_{B}, \zeta, \boldsymbol{P}_{h \perp}^{2}\right)} \\
& {\left[F_{L T}^{\cos \left(\phi_{h}-\phi_{S}\right)}\right]_{\mathrm{TFR}}=\sum_{a} e_{a}^{2} x_{B} \frac{\left|\boldsymbol{P}_{h \perp}\right|}{m_{h}} \Delta M_{T}^{h}\left(x_{B}, \zeta, \boldsymbol{P}_{h \perp}^{2}\right) .}
\end{aligned}
$$

For comparison, we recall that in the CFR the two structure functions above are given by [29]:

$$
\left[F_{U T, T}^{\sin \left(\phi_{h}-\phi_{S}\right)}\right]_{\mathrm{CFR}}=\mathcal{C}\left[-\frac{\hat{\boldsymbol{h}} \cdot \boldsymbol{k}_{\perp}}{m_{N}} f_{1 T}^{\perp} D_{1}\right], \quad\left[F_{L T}^{\cos \left(\phi_{h}-\phi_{S}\right)}\right]_{\mathrm{CFR}}=\mathcal{C}\left[\frac{\hat{\boldsymbol{h}} \cdot \boldsymbol{k}_{\perp}}{m_{N}} g_{1 T} D_{1}\right]
$$

where $\hat{\boldsymbol{h}} \equiv \boldsymbol{P}_{h \perp} /\left|\boldsymbol{P}_{h \perp}\right|$ and $\mathcal{C}$ denotes the transverse momentum convolution

$$
\begin{gathered}
\mathcal{C}[w f D] \equiv \sum_{a} e_{a}^{2} x_{B} \int \mathrm{d}^{2} \boldsymbol{k}_{\perp} \int \mathrm{d}^{2} \boldsymbol{\kappa}_{\perp} \delta^{2}\left(\boldsymbol{k}_{\perp}-\boldsymbol{\kappa}_{\perp}-\boldsymbol{P}_{h \perp} / z\right) \\
\times w\left(\boldsymbol{k}_{\perp}, \boldsymbol{\kappa}_{\perp}\right) f\left(x_{B}, \boldsymbol{k}_{\perp}^{2}\right) D\left(z_{h}, \boldsymbol{\kappa}_{\perp}^{2}\right) .
\end{gathered}
$$

\subsection{Lepto-production of a spinless hadron and a current jet}

Suppose now that the current quark jet is observed in coincidence with a spinless hadron in the TFR. In this case the cross section is differential both in $\boldsymbol{P}_{h \perp}$ and in $\boldsymbol{k}_{\perp}$. Thus, there is no $\boldsymbol{k}_{\perp}$ integration in Eqs. (49, 50). The final expression for the cross section is (for simplicity we omit the dependence of fracture functions on the variables $\left.x_{B}, \boldsymbol{k}_{\perp}^{2}, \zeta, \boldsymbol{P}_{h \perp}^{2}, \boldsymbol{k}_{\perp} \cdot \boldsymbol{P}_{h \perp}\right)$

$$
\frac{\mathrm{d} \sigma^{\mathrm{TFR}}}{\mathrm{d} x_{B} \mathrm{~d} y \mathrm{~d} \zeta \mathrm{d}^{2} \boldsymbol{P}_{h \perp} \mathrm{d}^{2} \boldsymbol{k}_{\perp} \mathrm{d} \phi_{S}}=\frac{2 \alpha_{\mathrm{em}}^{2}}{Q^{2} y}\left\{\left(1-y+\frac{y^{2}}{2}\right)\right.
$$




$$
\begin{aligned}
& \times \sum_{a} e_{a}^{2}\left[\hat{M}+S_{\|} \frac{\left|\boldsymbol{P}_{h \perp}\right|\left|\boldsymbol{k}_{\perp}\right|}{m_{h} m_{N}} \hat{M}_{L}^{\perp h} \sin \left(\phi_{h}-\phi_{j}\right)\right. \\
& \left.-\left|\boldsymbol{S}_{\perp}\right| \frac{\left|\boldsymbol{P}_{h \perp}\right|}{m_{h}} \hat{M}_{T}^{h} \sin \left(\phi_{h}-\phi_{S}\right)-\left|\boldsymbol{S}_{\perp}\right| \frac{\left|\boldsymbol{k}_{\perp}\right|}{m_{N}} \hat{M}_{T}^{\perp} \sin \left(\phi_{j}-\phi_{S}\right)\right] \\
& +\lambda_{l} y\left(1-\frac{y}{2}\right) \sum_{a} e_{a}^{2}\left[\frac{\left|\boldsymbol{P}_{h \perp}\right|\left|\boldsymbol{k}_{\perp}\right|}{m_{h} m_{N}} \Delta \hat{M}^{\perp h} \sin \left(\phi_{h}-\phi_{j}\right)+S_{\|} \Delta \hat{M}_{L}\right. \\
& \left.\left.+\left|\boldsymbol{S}_{\perp}\right| \frac{\left|\boldsymbol{P}_{h \perp}\right|}{m_{h}} \Delta \hat{M}_{T}^{h} \cos \left(\phi_{h}-\phi_{S}\right)+\left|\boldsymbol{S}_{\perp}\right| \frac{\left|\boldsymbol{k}_{\perp}\right|}{m_{N}} \Delta \hat{M}_{T}^{\perp} \cos \left(\phi_{j}-\phi_{S}\right)\right]\right\}
\end{aligned}
$$

where $\phi_{j}$ is the azimuthal angle of the jet. The situation is richer than in the previous process, but even in this case some of the modulations appearing in the production of a single hadron in the CFR, e.g. $\sin \left(\phi_{h}+\phi_{S}\right)$, are absent since we do not "measure" the final quark transverse polarization. From an experimental viewpoint, the main practical problem is that it is rather difficult to detect the quark jet.

\subsection{Lepto-production of a polarized hadron integrated over transverse momenta}

We have assumed so far that the final hadron is spinless or unpolarized. Relaxing this condition, the panorama of unintegrated fracture functions becomes extremely complicated. However, the collinear case, that is, the lepto-production of a polarized hadron integrated over all transverse momenta, is still manageable. Considering only the leading terms in the expansion of the traced fracture matrix we have (the superscript denotes the polarization state of the final hadron)

$$
\begin{aligned}
\int \mathrm{d}^{2} \boldsymbol{P}_{h \perp} \int \mathrm{d}^{2} \boldsymbol{k}_{\perp} \mathcal{M}^{\left[\gamma^{-}\right]} & =M\left(x_{B}, \zeta\right)+S_{\|} S_{h \|} M_{L}^{L}\left(x_{B}, \zeta\right)+\left(\boldsymbol{S}_{\perp} \cdot \boldsymbol{S}_{h \perp}\right) M_{T}^{T}\left(x_{B}, \zeta\right) \\
\int \mathrm{d}^{2} \boldsymbol{P}_{h \perp} \int \mathrm{d}^{2} \boldsymbol{k}_{\perp} \mathcal{M}^{\left[\gamma^{-} \gamma_{5}\right]} & =S_{\|} \Delta M_{L}\left(x_{B}, \zeta\right)+S_{h \|} \Delta M^{L}\left(x_{B}, \zeta\right)+\left(\boldsymbol{S}_{\perp} \times \boldsymbol{S}_{h \perp}\right) \Delta M_{T}^{T}\left(x_{B}, \zeta\right) .
\end{aligned}
$$

We see that an unpolarized target can emit a longitudinally polarized quark and a longitudinally polarized hadron, via $\Delta M^{L}$, a longitudinally polarized target can emit an unpolarized quark and a longitudinally polarized hadron, via $M_{L}^{L}$, and a transversely polarized target can produce a transversely polarized hadron in two different ways: via a correlation of the type $\boldsymbol{S}_{\perp} \cdot \boldsymbol{S}_{h \perp}$ by emitting an unpolarized quark, or via a correlation of the type $\boldsymbol{S}_{\perp} \times \boldsymbol{S}_{h \perp}$ by emitting a longitudinally polarized quark.

From Eqs. (57, 58) and (49, 50) we derive the cross section for the process $l N \rightarrow l^{\prime} h^{\uparrow} X$ :

$$
\begin{aligned}
& \frac{\mathrm{d} \sigma^{\mathrm{TFR}}}{\mathrm{d} x_{B} \mathrm{~d} y \mathrm{~d} \zeta \mathrm{d} \phi_{S} \mathrm{~d} \phi_{S_{h}}}=\frac{\alpha_{\mathrm{em}}^{2}}{\pi Q^{2} y}\left\{\left(1-y+\frac{y^{2}}{2}\right)\right. \\
& \quad \times \sum_{a} e_{a}^{2}\left[M\left(x_{B}, \zeta\right)+S_{\|} S_{h \|} M_{L}^{L}\left(x_{B}, \zeta\right)+\left|\boldsymbol{S}_{\perp}\right|\left|\boldsymbol{S}_{h \perp}\right| M_{T}^{T}\left(x_{B}, \zeta\right) \cos \left(\phi_{S_{h}}-\phi_{S}\right)\right] \\
& \quad+\lambda_{l} y\left(1-\frac{y}{2}\right) \sum_{a} e_{a}^{2}\left[S_{\|} \Delta M_{L}\left(x_{B}, \zeta\right)+S_{h \|} \Delta M^{L}\left(x_{B}, \zeta\right)\right. \\
& \left.\left.\quad+\left|\boldsymbol{S}_{\perp}\right|\left|\boldsymbol{S}_{h \perp}\right| \Delta M_{T}^{T}\left(x_{B}, \zeta\right) \sin \left(\phi_{S_{h}}-\phi_{S}\right)\right]\right\} .
\end{aligned}
$$


The transverse polarization terms contain modulations of the type $\cos \left(\phi_{S_{h}}-\phi_{S}\right)$ and $\sin \left(\phi_{S_{h}}-\phi_{S}\right)$, the former involving unpolarized quarks, the latter longitudinally polarized ones. It is instructive to compare this result with the corresponding cross section for current fragmentation, which is

$$
\begin{aligned}
& \frac{\mathrm{d} \sigma^{\mathrm{CFR}}}{\mathrm{d} x_{B} \mathrm{~d} y \mathrm{~d} z_{h} \mathrm{~d} \phi_{S} \mathrm{~d} \phi_{S_{h}}}=\frac{\alpha_{\mathrm{em}}^{2}}{\pi Q^{2} y} \\
& \cdot\left\{\left(1-y+\frac{y^{2}}{2}\right) \sum_{a} e_{a}^{2}\left[f_{1}\left(x_{B}\right) D_{1}\left(z_{h}\right)+S_{\|} S_{h \|} g_{1}\left(x_{B}\right) G_{1}\left(z_{h}\right)\right]\right. \\
& \quad-(1-y)\left|\boldsymbol{S}_{\perp}\right|\left|\boldsymbol{S}_{h \perp}\right| \sum_{a} e_{a}^{2} h_{1}\left(x_{B}\right) H_{1}\left(z_{h}\right) \cos \left(\phi_{S}+\phi_{S_{h}}\right) \\
& \left.\quad+\lambda_{l} y\left(1-\frac{y}{2}\right) \sum_{a} e_{a}^{2}\left[S_{\|} g_{1}\left(x_{B}\right) D_{1}\left(z_{h}\right)+S_{h \|} f_{1}\left(x_{B}\right) G_{1}\left(z_{h}\right)\right]\right\},
\end{aligned}
$$

where $f_{1}, g_{1}$ and $h_{1}$ are the unpolarized, helicity and transversity distributions respectively, and $D_{1}$, $G_{1}, H_{1}$ are the corresponding fragmentation functions. In the CFR cross section, the transverse spin term comes from a tensor component of the quark correlation matrix and involves the transversity distribution.

\section{Conclusions and perspectives}

We have presented the formalism of polarized and transverse-momentum dependent fracture functions to describe SIDIS processes in the TFR. At leading twist, neglecting all contributions of order $1 / Q$, and considering only spinless or unpolarized final hadrons, we find sixteen fracture functions defined in Eqs. (26) -(28). They represent the combined distributions of initial quarks and final hadrons inside a nucleon; the quark takes part in the elementary interaction, carrying away (in the collinear limit) a fraction $x_{B}$ of the nucleon energy $E$, while the remnant of the nucleon, with energy $\left(1-x_{B}\right) E$ fragments into the observed final hadron $h$ with energy $\zeta E=z\left(1-x_{B}\right) E$. We have taken into account the transverse momenta of quarks, $\boldsymbol{k}_{\perp}$, and of the final hadron $\boldsymbol{P}_{h \perp}$, as well as the nucleon and quark polarization. The final hadron polarization has only been briefly discussed in the collinear, or fully integrated, case. The fracture functions are divided into three classes, referring, respectively, to unpolarized, longitudinally polarized and transversely polarized quarks.

These quantities are probed in the target fragmentation region of SIDIS. We have seen that when a single hadron is detected in the TFR only two classes of fracture functions can be measured, representing the distributions of unpolarized and longitudinally polarized quarks. The fracture functions related to the distribution of transversely polarized quarks are chiral-odd quantities and could only be accessed by coupling such fracture funtions to other chiral-odd functions, like the Collins fragmentation function. This could be done in SIDIS processes with the detection of two final hadrons, one in the TFR and one in the CFR and will be discussed in a separate paper.

The explicit parton-model expressions of the cross sections and angular distributions have been worked out in three cases. The first is the lepto-production of a single spinless hadron in the TFR: in such a case no information can remain on $\boldsymbol{k}_{\perp}$, which is integrated, and one remains with four $\boldsymbol{P}_{h \perp}$-dependent fracture functions. Two of them survive upon integration over $\boldsymbol{P}_{h \perp}$, leading to the 
ordinary, fully integrated, fracture functions (the unpolarized and the helicity fracture functions). The other two generate interesting $\sin \left(\phi_{h}-\phi_{S}\right)$ and $\cos \left(\phi_{h}-\phi_{S}\right)$ azimuthal modulations. These fracture functions are analogous to the Sivers and the $g_{1 T}^{\perp}$ TMDs, which, in the CFR, indeed generate the same azimuthal dependences. A careful separation of the TFR and the CFR, when analyzing such azimuthal asymmetries is crucial.

The second case considered requires that the current jet is detected, in addition to the hadron in the TFR. In such a case $\boldsymbol{k}_{\perp}$ is observed and the eight fracture functions of the first two classes are fully involved, having all possibilities of correlations between the transverse momenta (of the jet and of the hadron) and the target spin.

Finally, we have considered the lepto-production of a polarized hadron integrated (for obvious reasons of simplicity) over all transverse momenta. Such a process probes six fracture functions, four of which require the measurement of the final hadron polarization (and are different from those previously discussed).

Many possible further developments of this work can be envisaged. As already stated, the most obvious extension is the full study of the case in which two hadrons are produced, one in the TFR and one in the CFR. The handbag parton model diagram for such a process is shown in Fig. 2 (left). In the simple case in which both final hadrons are spinless it couples the full set of fracture functions to the two leading-twist fragmentation functions (the unpolarized and the Collins fragmentation functions). The chiral-odd nature of the Collins function allows to access the (chiral-odd) fracture functions describing the distributions of transversely polarized quarks, Eq. (28).

Another potentially very interesting process is, in $N-N$ interactions, the usual Drell-Yan production of a lepton pair, with, in addition, the detection of a hadron produced in one of two TFRs, as shown in Fig. 2 (right). Such a process couples the fracture functions with the collinear PDFs or the TMDs; for $p-p$ interactions one could consider the fracture functions for quarks and the CFR distribution functions for anti-quarks (in a proton). Even better, with $N-\bar{p}$ interactions one could only refer to quark distributions inside a proton.

Finally, but not less important, there is the whole field of the phenomenological applications of the formalism of polarized fracture functions. This requires modeling in a simple way the fracture functions and computing the relevant observables in the kinematics of the available SIDIS experiments (HERMES, COMPASS, JLAB). In particular, it is important to assess the role of the fracture functions in the measured Sivers asymmetries, which so much have influenced the exploration of the 3-dimensional momentum structure of the nucleon.

Some phenomenological studies to describe spin phenomena for single hadron production in the TFR of SIDIS were performed using Monte Carlo event generators. For example, in Refs. [30, 31 the longitudinal polarization of $\Lambda$ baryons produced in the TFR of SIDIS of polarized leptons off an unpolarized or longitudinally polarized target was modeled for the transverse momentum integrated case (as in Sec. 5.3). Some predictions for the Sivers-type modulation of unpolarized hadron production in the TFR of SIDIS off transversely polarized protons were presented in Ref. [32. However, these results were obtained by an event generator based on the Lund string model, which covers both the $x_{F}<0$ and the $x_{F}>0$ regions, but does not distinguish between the two different dynamical mechanisms of fracture functions and current fragmentation functions.

The fracture function formalism we considered here gives, for single hadron production in the TFR, clear and rather simple predictions for azimuthal asymmetries, Eq. (51). The eventual ex- 

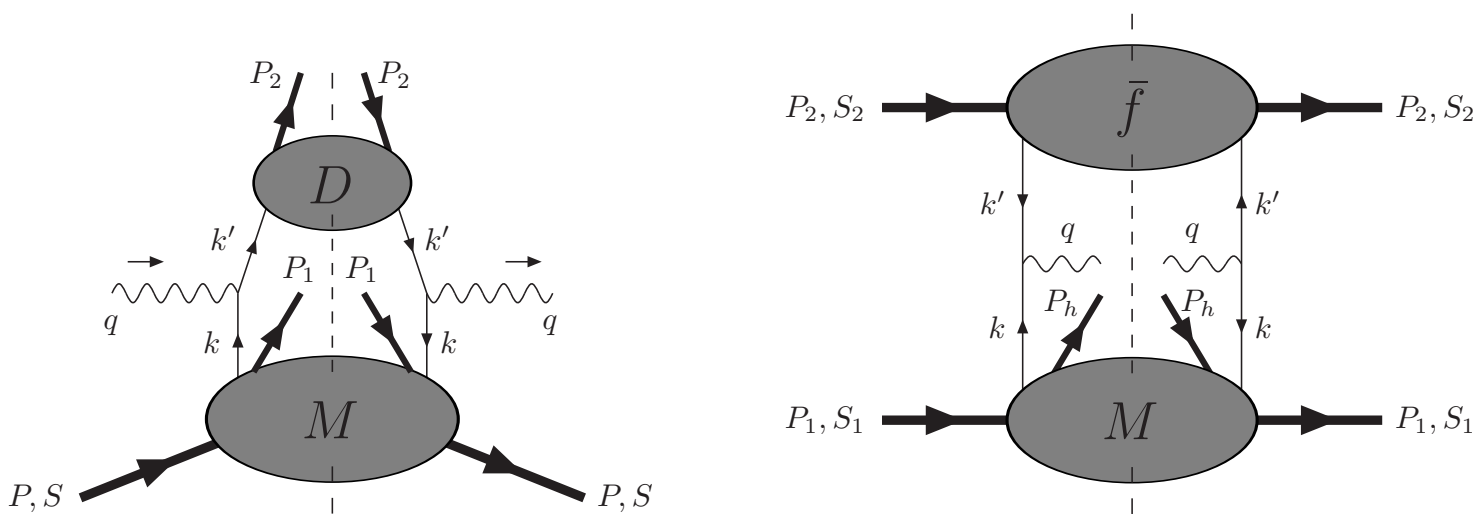

Figure 2: The handbag diagram for the lepto-production of two hadrons, one in the CFR and one in the TFR (left) and for the Drell-Yan production of a leading hadron (right).

perimental observation of other azimuthal modulations, for example a Collins-type $\sin \left(\phi_{h}+\phi_{S}\right)$ dependence, would indicate that the QCD fracture function approach, with a dynamical separation between the TFR and the CFR, does not hold and that long range correlations between the struck quark and the produced hadron might be important in the TFR as well.

The ideal experiments to test the fracture function factorization and measure these new functions in SIDIS, are those being discussed in the international community and planned at future Electron Ion or Electron Nucleon Colliders (EIC/ENC). The collider mode and the high energy would allow a clean kinematical separation between the TFR and the CFR and a thorough systematic and independent investigation of the two regions.

\section{Acknowledgements}

We thank Markus Diehl for useful discussions and for pointing out a redundancy in the list of fracture functions appearing in an earlier version of this manuscript. We acknowledge partial support by the Italian Ministry of Education, University and Research (MIUR) in the framework of a Research Project of National Interest (PRIN 2008). We also acknowledge partial support by the European Community - Research Infrastructure Activity under the FP7 program (HadronPhysics2, Grant agreement 227431), by the Helmholtz Association through funds provided to the Virtual Institute "Spin and Strong QCD"(VH-VI-231) and by Regione Piemonte.

\section{Appendix}

We exploit the following identities to perform the integration over $\boldsymbol{k}_{\perp}$ :

$$
\int \mathrm{d}^{2} \boldsymbol{k}_{\perp} k_{\perp}^{i} f\left(\boldsymbol{k}_{\perp}^{2}, \boldsymbol{P}_{h \perp}^{2}, \boldsymbol{k}_{\perp} \cdot \boldsymbol{P}_{h \perp}\right)=P_{h \perp}^{i} I_{1}^{[f]}\left(\boldsymbol{P}_{h \perp}^{2}\right),
$$




$$
\int \mathrm{d}^{2} \boldsymbol{k}_{\perp} k_{\perp}^{i} k_{\perp}^{j} f\left(\boldsymbol{k}_{\perp}^{2}, \boldsymbol{P}_{h \perp}^{2}, \boldsymbol{k}_{\perp} \cdot \boldsymbol{P}_{h \perp}\right)=P_{h \perp}^{i} P_{h \perp}^{j} I_{2}^{[f]}\left(\boldsymbol{P}_{h \perp}^{2}\right)+g^{i j} I_{3}^{[f]}\left(\boldsymbol{P}_{h \perp}^{2}\right),
$$

with

$$
\begin{aligned}
I_{1}^{[f]}\left(\boldsymbol{P}_{h \perp}^{2}\right) & =\int \mathrm{d}^{2} \boldsymbol{k}_{\perp} \frac{\boldsymbol{k}_{\perp} \cdot \boldsymbol{P}_{h \perp}}{\boldsymbol{P}_{h \perp}^{2}} f\left(\boldsymbol{k}_{\perp}^{2}, \boldsymbol{P}_{h \perp}^{2}, \boldsymbol{k}_{\perp} \cdot \boldsymbol{P}_{h \perp}\right), \\
I_{2}^{[f]}\left(\boldsymbol{P}_{h \perp}^{2}\right) & =\int \mathrm{d}^{2} \boldsymbol{k}_{\perp} \frac{2\left(\boldsymbol{k}_{\perp} \cdot \boldsymbol{P}_{h \perp}\right)^{2}-\boldsymbol{P}_{h \perp}^{2} \boldsymbol{k}_{\perp}^{2}}{\left(\boldsymbol{P}_{h \perp}^{2}\right)^{2}} f\left(\boldsymbol{k}_{\perp}^{2}, \boldsymbol{P}_{h \perp}^{2}, \boldsymbol{k}_{\perp} \cdot \boldsymbol{P}_{h \perp}\right), \\
I_{3}^{[f]}\left(\boldsymbol{P}_{h \perp}^{2}\right) & =\int \mathrm{d}^{2} \boldsymbol{k}_{\perp} \frac{\boldsymbol{P}_{h \perp}^{2} \boldsymbol{k}_{\perp}^{2}-\left(\boldsymbol{k}_{\perp} \cdot \boldsymbol{P}_{h \perp}\right)^{2}}{\boldsymbol{P}_{h \perp}^{2}} f\left(\boldsymbol{k}_{\perp}^{2}, \boldsymbol{P}_{h \perp}^{2}, \boldsymbol{k}_{\perp} \cdot \boldsymbol{P}_{h \perp}\right) .
\end{aligned}
$$

When deriving the momentum sum rules we use the analogous identities obtained by exchanging $\boldsymbol{k}_{\perp}$ with $\boldsymbol{P}_{h \perp}$.

\section{References}

[1] V. Barone, F. Bradamante and A. Martin, Progr. Part. Nucl. Phys. 65 (2010) 267, arXiv:1011.0909 [hep-ph].

[2] L. Trentadue and G. Veneziano, Phys. Lett. B323 (1994) 201.

[3] E.L. Berger, Argonne preprint ANL-HEP-CP-87-45.

[4] D. Graudenz, Nucl. Phys. B432 (1994) 351, hep-ph/9406274.

[5] D. de Florian and R. Sassot, Phys. Rev. D56 (1997) 426, hep-ph/9703228.

[6] M. Diehl and S. Sapeta, Eur. Phys. J. C41 (2005) 515, hep-ph/0503023.

[7] P.J. Mulders and R.D. Tangerman, Nucl. Phys. B461 (1996) 197, hep-ph/9510301.

[8] V. Barone, A. Drago and P.G. Ratcliffe, Phys. Rep. 359 (2002) 1, hep-ph/0104283.

[9] A.V. Belitsky, X. Ji and F. Yuan, Nucl. Phys. B656 (2003) 165, hep-ph/0208038.

[10] C.J. Bomhof, P.J. Mulders and F. Pijlman, Eur. Phys. J. C47 (2006) 147, hep-ph/0601171.

[11] M. Grazzini, L. Trentadue and G. Veneziano, Nucl. Phys. B519 (1998) 394, hep-ph/9709452.

[12] D. Sivers, Phys. Rev. D79 (2009) 085008, arXiv:0811.2388 [hep-ph].

[13] D. Sivers, Phys. Rev. D81 (2010) 034029, arXiv:0910.5420 [hep-ph].

[14] D. Sivers, Phys. Rev. D41 (1990) 83.

[15] M. Anselmino and F. Murgia, Phys. Lett. B442 (1998) 470, hep-ph/9808426.

[16] J.P. Ralston and D.E. Soper, Nucl. Phys. B152 (1979) 109. 
[17] A.M. Kotzinian, Nucl. Phys. B441 (1995) 234, hep-ph/9412283.

[18] R.D. Tangerman and P.J. Mulders, Phys. Lett. B352 (1995) 129, hep-ph/9501202.

[19] F.A. Ceccopieri and L. Trentadue, Phys. Lett. B636 (2006) 310, hep-ph/0512372.

[20] F.A. Ceccopieri and L. Trentadue, Phys. Lett. B660 (2008) 43, arXiv:0706.4242 [hep-ph].

[21] J.C. Collins, Phys. Rev. D57 (1998) 3051, hep-ph/9709499.

[22] J.C. Collins and D.E. Soper, Nucl. Phys. B194 (1982) 445.

[23] J. Levelt and P.J. Mulders, Phys. Rev. D49 (1994) 96, hep-ph/9304232.

[24] D. Boer and P.J. Mulders, Phys. Rev. D57 (1998) 5780, hep-ph/9711485.

[25] V. Barone, A. Prokudin and B.-Q. Ma, Phys. Rev. D78 (2008) 045022, arXiv:0804.3024 [hep-ph].

[26] V. Barone, S. Melis and A. Prokudin, Phys. Rev. D81 (2010) 114026, arXiv:0912.5194 [hep-ph].

[27] J.C. Collins, Nucl. Phys. B396 (1993) 161, hep-ph/9208213.

[28] M. Anselmino et al., Phys. Rev. D75 (2007) 054032, hep-ph/0701006.

[29] A. Bacchetta et al., JHEP 02 (2007) 093, hep-ph/0611265.

[30] J. R. Ellis, D. Kharzeev and A. Kotzinian, Z. Phys. C 69, 467 (1996) arXiv:hep-ph/9506280.

[31] J. R. Ellis, A. Kotzinian and D. V. Naumov, Eur. Phys. J. C 25, 603 (2002) arXiv:hep-ph/0204206.

[32] A. Kotzinian, arXiv:hep-ph/0504081. 